\title{
La calidad de vida según la vivencia de los mayores dependientes y sus cuidadores
}

The quality of life according to the experience of dependent elderly and their caregivers

\section{Montserrat Puig-Llobet ${ }^{1}$, Carmen Ferre-Grau ${ }^{2}$, Nuria Rodríguez-Ávila ${ }^{3}$, Teresa Lluch-Canut ${ }^{4}$, Juan Roldan-Merino ${ }^{5}$, Carmen Moreno-Arroyo ${ }^{6}$}

\author{
'Departamento de Enfermería de Salud Pública, Salud Mental y Materno-infantil. Universidad de Barcelona. Barcelona, España. \\ ${ }^{2}$ Departamento de Enfermería. Universidad Rovira i Virgili. Tarragona, España. \\ ${ }^{3}$ Departamento de Sociología y Análisis de las Organizaciones. Universidad de Barcelona. Barcelona, España. \\ ${ }^{4}$ Departamento de Enfermería de Salud Pública, Salud Mental y Materno-Infantil. Universidad de Barcelona. Barcelona, España. \\ ${ }^{5}$ Campus Docent Sant Joan de Déu. Escuela Universitaria Enfermería de San Joan de Déu. Barcelona, España. \\ ${ }^{6}$ Departamento de Enfermería Fundamental y Médico-Quirúrgica. Universidad de Barcelona. Barcelona, España. \\ Contacto: Montserrat Puig-Llobet / monpuigllob@ub.edu \\ Fecha de recepción: 17 de mayo de 2017 / Fecha de aceptación: 9 de agosto de 2017
}

\begin{abstract}
Resumen
Introducción: La calidad de vida es difícil de definir y operativizar al ser un concepto muy amplio, complejo y pluridimensional. El objetivo fue comparar las definiciones, las percepciones y las dimensiones relacionadas con la calidad de vida proporcionadas por las personas mayores dependientes y cuidadores.

Metodología: de tipo cualitativo a partir del estudio de caso. Las dimensiones estudiadas estaban relacionadas con la calidad de vida y aspectos sociodemográficos, de salud, dependencia y laborales según la población de estudio. La muestra final fue de 26 personas mayores, 22 cuidadores informales y 11 enfermeras del Servicio de Atención Domiciliaria del Centro de Atención Primaria de Vilafranca del Penedès.

Resultados: se encontraron elementos comunes en las definiciones, la percepción y elementos determinantes de la CV con algunos factores distintos según la población de estudio.
\end{abstract}

Palabras clave: calidad de vida, personas mayores dependientes, cuidadores informales.

\begin{abstract}
Introduction: Quality of life is difficult to define and operationalize being a very broad, complex and multidimensional concept. The aim was to compare the definitions, perceptions and dimensions related to the quality of life by the dependent elderly and caregivers.

Methodology: qualitative case study type. The dimensions studied were related to quality of life and sociodemographic, health, and labor dependence according to the study population. The final sample was 26 elderly, 22 informal caregivers and 11 nurses Home Care Service Center Primary Care Vilafranca.

Results: Common elements in definitions, perception and determinants of Quality of life with some factors other according to the study population.
\end{abstract}

Keywords: quality of life, elderly dependents, informal caregivers.

\section{Introducción}

La calidad de vida es difícil de definir y operativizar al ser un concepto muy amplio, complejo y pluridimensional, aplicado a muchos ámbitos de la vida cotidiana y muchas veces utilizado de forma imprecisa o equivocada. Existen distintos modelos que intentan relacionar los elementos implicados en la calidad de vida. Destaca el modelo de World Health Organitation Qua- lity of Life (WHOQOL-100), que consta de seis áreas de valoración de calidad de vida (CV): la salud física, el estado psicológico, el nivel de independencia, las relaciones sociales, el ambiente y las creencias personales. Su objetivo es que pueda utilizarse en distintas culturas, en diferentes problemas de salud y en situaciones especiales como por ejemplo, ser cuidador o ser una persona mayor dependiente ${ }^{1,2}$. Cabe destacar algunos modelos anglosajones que son construidos a partir 
de las informaciones de las personas mayores sobre los aspectos que consideran importantes en su calidad de vida. Dichos modelos, ponen en evidencia que los mayores comparten elementos comunes en la forma de entender y percibir la calidad de vida ${ }^{2}$.

Otro estudio realizado en cinco países europeos: Alemania, Italia, Países Bajos, Suecia y Reino Unido, establece unas áreas útiles en la valoración de la calidad de vida de los mayores: definición y valoración de la calidad de vida, entorno (barrio, vivienda, transporte y nuevas tecnologías), salud física y psíquica, apoyo familiar y social, empleo y ingresos, desigualdades, participación e integración social, papel de los servicios, satisfacción con la vida y bienestar subjetivo ${ }^{3}$.

Por último destacamos el modelo multidimensional de Fernández-Ballesteros (1992), que explica la calidad de vida de la persona mayor, a partir de dos clasificaciones interrelacionadas, distinguiendo las dimensiones personales de las dimensiones socioambientales y diferenciando a su vez los elementos subjetivos y los objetivos que la constituyen. Valorando factores personales como la salud, la autonomía y la satisfacción, así como factores socioambientales como las redes de apoyo o los servicios sociales ${ }^{4}$. En esta línea, Sequeria (2010) $)^{5}$ expone que en situaciones de dependencia aumenta la necesidad de ayuda en las actividades de la vida diaria y el mantenimiento de la calidad de vida.

Tal como se ha descrito, existen muchos modelos que intentan exponer los elementos relacionados con la calidad de vida, sin embargo no existe ninguno que intente determinar: “¿Qué elementos participan en la calidad de vida de las personas mayores dependientes de 75 y más años y sus cuidadores familiares atendidos por el servicio domiciliario ATDOM?"; por ello el objetivo del estudio fue comparar las definiciones, las percepciones y las dimensiones relacionadas con la calidad de vida en los actores implicados en situación de dependencia.

\section{Metodología}

El ámbito de estudio fue el Centro de Atención Primaria de l'Alt Penedès (Barcelona, Spain). La metodología utilizada fue de tipo cualitativo a partir del estudio de caso.

La población de estudio fueron las personas mayores dependientes de 75 y más años atendidos por el
Servicio de Atención Domiciliaria (ATDOM), sus cuidadores informales y las enfermeras del servicio ATDOM. Después de pasar por el Comité Ético del Instituto Catalán de Salud se nos proporcionó la lista de la población de estudio para poder obtener la muestra, a partir de la lista se eligieron las personas mayores que cumplían las siguientes criterios de inclusión: tuvieran un cuidador informal principal, no tuvieran ninguna enfermedad cognitiva y quisieran participar en el estudio. El muestreo fue intencionado, eligiendo a 26 mayores que representaban todos los estratos (por grupo de edad y género), ayudados por las enfermeras del servicio ATDOM. En relación a la elección de los cuidadores familiares, se utilizó un criterio estratégico, entrevistando a los cuidadores principales de los mayores entrevistados, dando su consentimiento un total de 22 cuidadores familiares. Respecto a las enfermeras del servicio ATDOM, se entrevistó a todas las enfermeras que hiciera al menos dos años que estaban en el servicio, un total de 11 .

\section{Variables y Dimensiones del estudio}

En la persona mayor y el cuidador informal principal se recogieron variables sociodemográficas, familiares y de salud; en la persona mayor también se identificó el nivel de dependencia y en el cuidador informal el nivel de sobrecarga.

Las dimensiones estudiadas fueron la definición de calidad de vida en los mayores dependientes, los cuidadores principales y las enfermeras; la percepción de calidad de vida y sus elementos determinantes en los mayores y cuidadores, obteniendo también la visión de las enfermeras respecto a la definición de calidad de vida y la percepción de esta de los mayores y cuidadores informales a los que atiende.

Una vez obtenido el permiso se realizó una reunión informativa con todas las enfermeras del servicio ATDOM del Área de salud Alt Penedès para explicarles el procedimiento. Una vez elegidas las personas a entrevistar, las enfermeras llamaron a sus pacientes para pedir permiso e informar a los mayores que la investigadora principal del estudio iría a su domicilio para entrevistarlos a ellos y a su cuidador principal. La técnica utilizada fue una entrevista cara a cara a partir de un formulario semiestructurado ad hoc y un guión de entrevista semiestructurada, con una duración de 45 minutos cada entrevista y grabada previo consentimiento. Se entrevistó por separado a las personas mayores y a sus cuidadores para evitar sesgos. Las entrevis 
tas a las enfermeras se realizaron en sus consultas.

En el análisis de datos, por un lado se realizó un análisis descriptivo utilizando frecuencias y porcentajes para las variables cualitativas, así como la media para algunas variables cuantitativas, por otro lado para el análisis cualitativo se transcribieron las entrevistas y se agruparon las dimensiones por categorías, codificándolas y relacionándolas por temas.

\section{Resultados}

\section{Características de la muestra}

Las personas mayores del estudio no presentaban síntomas de deterioro cognitivo, tenían un nivel de dependencia moderada según el índice de Barthel. La mayoría necesitaban ayuda para bañarse o ducharse, caminar o movilizarse según el índice de Katz y realizar la comida y las compras según el índice de Lawton. Las principales patologías que padecían eran las músculo esqueléticas, las cardiovasculares, las endocrinas, las pulmonares y las digestivas, y el $89 \%$ (n:19) de los mayores tenían pruripatología y tomaba más de una medicación. Los cuidadores familiares eran en su mayoría mujeres, con una media de edad de 64 años. La distribución por género mostró que las mujeres representan el 72,7\% del total de los cuidadores familiares. Se observó que, en primer lugar, eran las hijas las principales cuidadoras, siendo los cónyuges los que ocupan el segundo lugar en la realización de los cuidados principales, en tercer lugar los hijos y en último lugar otros familiares, y cuidaban una media de 7 horas diarias. Presentaban déficit de autocuidado y sobrecarga en cuidados aunque no lo reconocían y el test de Zarit decía lo contrario. Algunos tenían más de una enfermedad crónica y tomaban más de una medicación. La mayoría no trabajaba o estaba jubilada. En cuanto a las características de los once profesionales enfermeros entrevistados, se señala que trabajaban en el Área Básica de Salud de Vilafranca del Penedès y tenían una media de edad de 50 años, siendo la mayoría mujeres. Su jornada laboral era de 36 horas semanales, distribuidas aproximadamente en unas 6,30 horas de trabajo diarias, de las cuales una hora al día se dedicaba al servicio de Atención Domiciliaria (programa ATDOM). La formación de estos profesionales es de carácter universitario, con titulación de primer ciclo; algunos tienen formación en Geriatría y otros en Salud Pública. Están preocupados por su formación continuada, aunque la mayoría no tiene una formación especializada en personas mayores.

\section{Dimensiones calidad de vida}

\section{Definiciones CV de la Persona mayor}

Salud: "Tener salud es lo más importante" (E.01, E.06, E.15, E.16, E.17, E.18).

Salud y autonomía: "Tener salud y autonomía" (E.09, E.10).

Salud y recursos: "Me cuesta decirlo, a lo mejor tener más dinero (para arreglo, ropa), pero la salud, la salud es lo principal de todo" (E.03); "Tener salud, poder caminar" (E.08); "Tener salud y dinero" (E.12); "Tener salud y compañía” (E.21, E.24); “Tener salud y ayuda” (E.23); "Tener 50 años menos, ser más joven y poder trabajar (risas), me gustaría llevar un camión de basura. Que toda la familia tenga salud”(E.13).

Amor y otros: "Estar acompañada, no estar sola" (E.02); "Tener amor" (E.26); "Tener amor, cariño y buenas relaciones, sólo”(E.22).

Relaciones familiares: "Vivir bien en casa, tener buenas relaciones" (E.04); "Buenas relaciones con la familia” (E.14); "Autorrealización, porque quería ser cantante y no me dejaron y tener mejores relaciones" (E.11).

Adaptarse: "Conformarte" (E.07); "Tener salud y adaptarse” (E.20).

Vida social: “Tener saludy compañia”"(E.21, E.24).

\section{Definiciones de CV del Cuidador informal}

Salud: “Tener salud" (E.01, E.02, E.07, E.09, E.10, E.13, E.14).

Salud y autonomía: "Tener salud y autonomía" (E.12); "Independenciay salud" (E.22).

Salud y recursos: Tener salud, recursos y buenas relaciones" (E.03); "Hacer lo que uno quiere, tener salud y recursos" (E.19); "Tener salud y trabajo" (E.21).

Amor y otros: "Saludy amor" (E.08); "Tener salud, dinero y amor" (E.06); "Tener salud, amor y recursos" (E.11).

Relaciones familiares: "Tener salud y buenas relaciones familiares” (E.20).

Adaptarse: "Conformarte con la situación actual, adaptación” (E.05); "Estar a gusto con uno mismo, poder adaptarse a las nuevas situaciones" (E.15); “Adaptarse a la vida, a la familia”"(E.17).

Vida social: "Hacer lo que uno quiere, poder salir" (E.16).

Tiempo: "Tener tiempo para disfrutar y estar bien con uno mismo" (E.04); "Independencia y autonomía" (E.18). 


\section{Definiciones $C V$ de las enfermeras}

Salud: "Tener salud para poder hacer una vida normal" (E06).

Salud y autonomía: "Es vivir de forma independiente y autónoma" (E04); "Tener saludy autonomía"(E05). Satisfacción con la vida: "Es que la persona sea feliz e independiente" (E01); "Es una sensación subjetiva, agradable y multifactorial" (E02); "Es vivir satisfactoriamente"(E03).

Bienestar bio-psico-social: "Completo bienestar físico, psíquico y social" (E07); "Estado de bienestar físico y psíquico en el día a día" (E08); "Estar bien con uno mismo y los demás" (E09); "Cubrir las necesidades y estar bien con uno mismo de forma fisica y mental" (E10); "Salud fisica, psíquica y social y tener alguien para poder relacionarte"(E11).

A las personas mayores y a los cuidadores informales les resultó difícil y complicado establecer una definición de calidad de vida, habiendo de realizar un esfuerzo enorme para conseguir expresar una definición del concepto. Parte de la dificultad se debe a la complicación que existe en la definición del constructo. En cambio, en las definiciones de los profesionales fue muy fácil y en ellas se muestra la influencia de la disciplina enfermera, identificando el ser humano desde unas perspectiva holística. Entre las respuestas realizadas por los mayores entrevistados, se determina que definen el estado de salud como elemento necesario en la calidad de vida. Sin embargo, mientras unos remarcan la salud de forma exclusiva, otros la incorporaron junto a otros conceptos como tener autonomía, tener compañía, tener ayuda en su vida diaria, adaptarse a la situación actual o tener dinero y amor. En cuanto a los cuidadores familiares, también remarcan la salud como elemento destacado de su definición de calidad de vida; en algunas definiciones se expresó de esta forma exclusiva y en otras se incorporó junto a otros conceptos: tener dinero y recursos, tener buenas relaciones, tener amor, tener autonomía y tener trabajo. Se observan las diferencias entre los elementos relacionados con la CV según los entrevistados. Las enfermeras incorporan los aspectos de bienestar biopsicosocial, los mayores, como dice la canción, "salud, dinero y amor" y los cuidadores informales incorporan además el tener tiempo para ellos.

\section{Percepción de calidad de vida}

Respecto a la percepción de la calidad de vida en los mayores de 75 años con dependencia, se observa que la mayoría expresan tener una buena calidad de vida. Al determinar la percepción de calidad de vida de los cuidadores informales, la respuesta más frecuente fue de buena calidad de vida y ninguno la percibió como mala.

En la tabla 1 se detalla la frecuencia y \% respecto a la percepción de calidad de vida en los mayores y en los cuidadores informales.

\begin{tabular}{|lcccc|}
\hline Percepción CV & \multicolumn{2}{c}{ Mayores } & \multicolumn{2}{c|}{ Cuidadores } \\
& $\mathbf{\%}$ & $\mathbf{n}$ & $\mathbf{\%}$ & $\mathbf{n}$ \\
Muy buena & 0 & 0 & 4.5 & 1 \\
Buena & 76.9 & 20 & 50 & 11 \\
Regular & 15.4 & 4 & 45.5 & 10 \\
Mala & 7.7 & 2 & -- & - \\
Total & 100 & & 100 & \\
& $\mathrm{n}=26$ & & $\mathrm{n}=22$ \\
\hline
\end{tabular}

Tabla 1: Percepción CV mayores y cuidadores informales

Se observa que en comparación hay más mayores que cuidadores informales que perciben una buena calidad de vida, los profesionales también dijeron que algunos mayores tenían mejor calidad de vida que su cuidador.

\section{Dimensiones relacionadas con la calidad de vida}

Las personas mayores determinan como dimensiones relacionadas con una buena CV la salud, adaptación, los recursos, el amor, la autonomía y el apoyo familiar, en cambio los cuidadores solo la salud, la adaptación y el tiempo. Relacionan con tener una mala o regular percepción de calidad de vida la pérdida de autonomía y salud, la soledad y la aceptación.

Los cuidadores informales relacionan el tener salud, tiempo para el ocio y la vida social y adaptarse a la situación, como determinantes de la calidad de vida. Los motivos relacionados con la percepción de una determinada calidad de vida están relacionados principalmente con estar satisfecho y contento de ser cuidador, le sigue tener salud, tener buenas relaciones y tener recursos sociosanitarios. Los motivos relacionados con una regular o mala percepción de la calidad de vida son por estar muy atados, tener poca salud y tener pocas relaciones sociales. Se aprecia una relación entre el estado de salud y la percepción de calidad de vida. Se determina que a menos salud del cuidador familiar y del mayor al que cuidan, peor percepción de calidad de vida presentan los cuidadores familiares. En relación a 
Factores

Adaptación

\section{Personas mayores}

"Buena, me he adaptado a no poder

caminar, a no bajar las escaleras" (E.07).

"Buena, porque me adapto a las necesidades" (E.08).

"Buena, estoy bien por la edad que tengo y estoy cuidada" (E.12).

"Buena, estoy bien por la edad que tengo, no puedo pedir más" (E.13).

"Buena, adaptada a la situación, leo mucho" (E.16).

"Buena, porque a mi edad, tengo un poco de salud, dinero y amor" (E.19).

"Buena, uno ha de adaptarse a las circunstancias, vaya creo que sí" (E.26).

"Buena, porque no me falta de nada, entre

Recursos unos yotros me ayudan" (E.04).

"Buena, porque no me falta nada, tengo recursos y salud, puedo comprar lo que quiero" (E.05).

"Buena, porque tengo todo lo que quiero, aunque no mucha salud" (E.11).

"Buena, no me falta de nada" (E.14).

"Buena, porque no me falta de nada, tengo todo lo que necesito, hombre me gustaría caminar" (E.20).

Salud "Buena, porque tengo salud y compañia"
(E.02)
"Yo me siento bien, es buena" (E.03)
"Buena, ahora si, me encontraba mal antes,
pero ahora bien, pensaba mal, estaba
asustada (E.15).

"Buena, porque me encuentro bien" (E.24).

\section{Cuidadores}

"Buena porque acepto la situación que tengo" (E.05, E.06).

“Buena si me conformo y adapto"(E.03, E.04).
"Buena cuando se tiene salud" (E.01, E.02, E.07, E.09).

"Buena, porque en casa hay amor" (E01).

\begin{tabular}{ll} 
Amor & "Buena, porque en casa hay amor" (E01). \\
\hline Autonomía & $\begin{array}{l}\text { "Buena, porque puedo valerme por mi } \\
\text { misma" (E.10). }\end{array}$ \\
\hline $\begin{array}{l}\text { Apoyo } \\
\text { familiar }\end{array}$ & $\begin{array}{l}\text { "Buena, porque me encuentro apoyado por } \\
\text { todos hijos" (E.22), "Buena, porque } \\
\text { estoy cuidado" (E.23). }\end{array}$ \\
\hline
\end{tabular}

Tiempo

"Tener tiempo para uno mismo" (E.08, E.10).

"Poder salir con los amigos y pareja" (E.11).

Tabla 2: Factores que los mayores y los cuidadores relacionan con una buena $\mathrm{CV}$ 
los problemas psicológicos que los cuidadores relacionan con su calidad de vida son la angustia, la depresión y el estrés. Las respuestas de los mayores y cuidadores respecto a los elementos relacionados con tener una buena percepción de calidad de vida se reflejan en la tabla 2 .

Si comparamos lo que consideran como calidad de vida los mayores y lo relacionamos en cómo perciben su estado actual, se observa que en las definiciones el elemento identificado con más frecuencia es la salud. En cambio, al preguntarles cómo determinan la calidad de vida actual, la adaptación a la situación actual tiene un peso importante en las respuestas. Esto explica que en la percepción de una determinada calidad de vida la capacidad de adaptación es un elemento importante a tener en cuenta.

\section{Discusión}

Se expresa la dificultad de definir el constructo calidad de vida en los mayores y en los cuidadores informales. Entre las respuestas realizadas por los mayores entrevistados, se determina que definen el estado de salud como elemento necesario en la calidad de vida y son varios los estudios que remarcan la importancia de la salud en la valoración de la calidad de vida ${ }^{1,2,3,6-12}$. Asimismo, otros elementos mencionados por los mayores respecto a tener una buena calidad de vida son: tener relaciones sociales, tener compañía, sentirse querido y mantener buenas relaciones. Diversos estudios determinan dichos elementos como importantes en la determinación de la calidad de vida ${ }^{2,4,7-10,12-14}$.

Referente a la percepción de la calidad de vida, la mayoría expresaron tener una buena calidad de vida, siendo sólo un 7,7\% $(n=2)$ las personas mayores que dicen tener una mala calidad de vida, datos observados en otros estudios ${ }^{2,10}$. En general, observamos que las mujeres perciben mejor calidad de vida que los hombres, sin embargo la existencia de más mujeres en la muestra puede producir sesgos en dichos resultados. Además, en otro estudio se muestra lo contrario, es decir, son los hombres los que generalmente perciben mejor calidad de vida ${ }^{2}$.

Al igual que en otros países, las mujeres son las que ejercen como cuidadoras, además muchas veces no perciben el nivel de sobrecarga real ${ }^{12,15-18}$. Al determinar la percepción de calidad de vida de los cuidadores, la respuesta más frecuente fue la de una buena calidad de vida. Si comparamos los resultados con el estudio de
Pérez (2006) ${ }^{19}$, observamos la misma tendencia. Algunos estudios también destacan como elementos relacionados con la percepción de una determinada calidad de vida: la falta de tiempo libre, la percepción de un mal estado de salud físico y psíquico, el tener una red de apoyo social, el tener relaciones familiares satisfactorias, el contar con amor y afecto y el estar satisfecho con el ambiente familiar ${ }^{19-21}$. En un estudio del IMSERSO (2005), se muestra que los elementos que más han modificado la vida del cuidador familiar son los aspectos relacionados con el ocio y tiempo libre para ellos mismos y el tiempo para conciliar su vida familiar $^{22}$. Algunos estudios destacan que es importante para los cuidadores tener servicios de apoyo o descanso parar evitar las sobrecarga de cuidados ${ }^{15,23}$.

Relacionamos la adaptación al rol social de las personas mayores dependientes según la perspectiva de rol del funcionalismo de Parsons ${ }^{24}$ y según la teoría de la desvinculación de Cumming y Henry ${ }^{25}$, que constata que el mayor se aleja poco a poco de la vida social y la sociedad también le ofrece cada vez menos posibilidades. De modo que no se contempla la necesidad de establecer contactos sociales a partir de cierta edad, ni por parte de los mayores ni por parte de la sociedad y por ello se adaptan a la situación que tienen. Respecto al cuidador, pensamos que dicha conciliación o adaptación se debe al rol y socialización que las mujeres de las generaciones implicadas en este estudio tienen respecto a su condición de cuidadoras. Por ello, ubicamos dichos resultados en la perspectiva teórica del funcionalismo de Parsons, que relaciona la socialización con la adquisición de determinados roles sociales, en este caso el de cuidadora. Las limitaciones del estudio son principalmente que el tamaño de la muestra no es representativa como ocurre con los estudios cualitativos y que el estudio incluye solamente a personas dependientes que son atendidas por los servicios públicos de salud de la ciudad, quedando por estudiar los pacientes que son atendidos por servicios privados. Otra limitación es que al ser la mayoría cuidadoras no permite ver cómo viven esta realidad los hombres que realizan los cuidados.

\section{Conclusiones}

Las definiciones de calidad de vida son muy similares en las personas mayores y sus cuidadores, aunque se destaca que en los cuidadores el tener tiempo libre es importante. 
La salud es un elemento clave en las definiciones y percepciones de la calidad de vida de las personas mayores y cuidadores, pero no el único.

La adaptación es muy importante para conseguir el bienestar.

La socialización del rol de cuidadora ayuda en la aceptación de ser cuidador y percibir una mejor calidad de vida, sin embargo ello hace que no expresen sus necesidades.

\section{Aplicabilidad}

El proporcionar ayuda a los cuidadores informales fomentando su autocuidado y sus espacios de respiro y tiempo libre puede ayudar a vivir con menos sobrecarga a estos cuidadores; para ello debe incluirse como estrategia clave en los planes de salud y atención primaria y domiciliaria, informando y creando los recursos sociales necesarios.

\section{Bibliografía}

1. Lucas, R. Versión española del WHOQOL. Madrid: Ediciones Ergon, 1998.

2. Fundació Viure i Conviure. Qualitat de vida de les persones grans a Catalunya. Barcelona: Caixa Catalunya. Obra Social, 2006

3. Walker A. Calidad de vida de las personas mayores. Análisis comparativo europeo. Rev Española Geriatr Gerontol. 2004; 39(3):8-17.

4. Fernández-Ballesteros, R. (1998) Calidad de vida: Las condiciones diferenciales. Colegio Oficial de Psicólogos 1998; 2. (1):57-65.

5. Sequeira, C. Cuidar de idosos com dependência física e mental. Lisboa, Portugal: Lidel, 2010.

6. Fernández-Ballesteros, R; Zamarrón, M.D; Maciá, A. Calidad de vida en la vejez en distintos contextos. Madrid: Ministerio de Trabajo y Asuntos sociales. IMSERSO, 1996.

7. Fernández-Ballesteros, R. Calidad de vida ¿es un concepto psicológico? Rev Española Geriatr Gerontol 2004; 39(3):18-22.

8. Sastre, MJ; Nyssen, JM; Furio, D; Durán, MC; Garrido, B; Toledo, A, “et al". Indicadores de calidad de vida. Un retrato del bienestar en España. Opiniones y actitudes. 47. Madrid: Centro de Investigaciones Sociológicas, 2003

9. Yanguas, JJ. Análisis de la calidad de vida relacionada con la salud en la vejez desde una perspectiva multidimensional. Madrid: IMSERSO, 2006

10. Fernández-Mayoralas, G; Rojo, F; Prieto, ME; León, B; Martínez, P; Forjaz, J. "et al". El significado de la salud en la calidad de vida de los mayores. 2007. Disponible en:

http://imsersomayores.csic.es/documentos/documentos/fern andezmayoralassignificado-01.pdf. [acceso 1 jul 2009].

11. Puig, M. Cuidados y Calidad de vida en Vilafranca del Penedès: Los mayores de 75 años o más, atendidos por el servicio de atención domiciliaria y sus cuidadores familiares. [tesis doctoral]. Barcelona: Facultad de Ciencias Económicas. Universidad de Barcelona, 2009.

12. Puig, M; Rodriguez, N; Lluch-Canut, MT; Moreno, C; Roldán, J; Montesó, P. Quality of life and care burden among informal caregivers of elderly dependents in Catalonia. Revista Portuguesa de Enfermagem de Saúde Mental [Internet]. 2015; (14):914.Disponible en:
<http://www.scielo.mec.pt/scielo.php?script=sci_arttext\&pid=S 1647-21602015000300002\&lng=pt> [acceso 11.3.2016].

13. Bowling A, Gabriel Z, Dykes J, Dowding LM, Evans O, Fleissig A, "et al". Let's ask them: a national survey of definitions of quality of life and its enhancement among people aged 65 and over. Int J Aging Human Develop 2003;56(4):269-306.

14. Melo R, Rua M, Vilaça de Brito, C. Necessidades do cuidador familiar no cuidado à pessoa dependente: uma revisão integrativa da literatura. Revista Enfermagem Referência 2014; 4(2):143151.

15. Cruz D. C. M, Loureiro H. A. M, Silva M. A. N. C. G. M. M, Fernandes M. M. (2010). As vivências do cuidador informal do idoso dependente. Revista de Enfermagem Referência 2010;3(2), 127-136.

16. van Ryn M, Sanders S, Kahn K, van Houtven C, Griffin J. M, Martin M, "et al". Objective burden, resources, and other stressors among informal cancer caregivers: a hidden quality issue? Psycho-Oncology.2011;20(1), 44-52

17. American Association of Retired Persons. Caregiving and long term care. Disponible en <www. research.aarp.org/health> [acceso: 22.03.2012].

18. Vaquiro Rodríguez S, Stiepovich Bertoni J. Cuidado informal, un reto asumido por la mujer. Ciencia y enfermería 2010; 2, 9-16.

19. Pérez, A. El cuidador primario de familiares con dependencia: Calidad de vida, apoyo social y salud mental. [tesis doctoral]. Salamanca: Facultad de Medicina, Universidad de Salamanca, 2006.

20. Carretero, S; Garcés, J; Ródenas, F.J;Sanjosé, V. La sobrecarga de las cuidadoras de personas dependientes, Análisis y propuestas de intervención psicosocial, Políticas de Bienestar Social. Valencia: Tirant lo Blanc, 2006.

21. Crespo, M; López, J. El apoyo a los cuidadores de familiares mayores dependientes en el hogar: Desarrollo del programa. Madrid. IMSERSO, 2007.

22. IMSERSO. Cuidados a las personas mayores en los hogares españoles: El entorno familiar. Colección Estudios Serie Dependencia. Madrid: IMSERSO, 2005.

23. Sotto Mayor M, Leite M. Implicações psicológicas da experiência informal de cuidar. Revista Portuguesa de Enfermagem de Saúde Mental 2011; 5,37-44

24. Parsons, T. El sistema Social. Madrid: Alianza, 1999

Cummings, E; Henry, W. Growing old: The process of disengagement. New York: Basic Books, 1961 